\title{
Probiotics on Intestinal Flora Disturbance and Bacterial Translocation in Mice with Inflammatory Bowel Disease
}

\author{
LIYING DING*, XIUTING CHEN, K. QIAN AND Z. JIANG \\ Department of Gastroenterology, Zhuji Hospital of Traditional Chinese Medicine, Zhuji, Zhejiang 311800, PR China
}

Ding et al.: Probiotics on Intestinal Flora Disturbance and Bacterial Translocation

\begin{abstract}
Inflammatory bowel disease is becoming more and more common in western countries and less common in China. However, with the change of dietary structure, the incidence of inflammatory bowel disease is also increasing in China. It will cause abdominal pain, diarrhea and mucus, pus and blood, which are repeatedly delayed and difficult to alleviate. To explore the effectiveness of probiotics in the treatment of inflammatory bowel disease, Lactobacillus plantarum in probiotics and fifteen 8 w old female mice were selected for the experiment. We divided the mice into three groups: blank control group, interleukin-10 group and interleukin-10+Lactobacillus plantarum group. The interleukin-10+Lactobacillus plantarum group was gavaged with $0.5 \mathrm{ml}$ of Lactobacillus plantarum bacterial solution $\left(1.0 \times 10^{9} \mathrm{CFU} / \mathrm{ml}\right)$ every d. The blank control group and interleukin-10 group were gavaged with $0.5 \mathrm{ml}$ of Ringer buffer for 4 consecutive w and compared the changes caused by Lactobacillus plantarum on Bifidobacterium, Lactobacillus, Clostridium perfringens and Enterobacter in the intestinal flora of mice and the changes caused by Lactobacillus plantarum on the intestinal flora effect of bacterial translocation in membranous lymph nodes and spleen. We found that the content of Enterobacter in the blank control group was the lowest at all three time points, with an average of 6 . The content of Enterobacter in the interleukin-10+Lactobacillus plantarum group and the interleukin-10 group was 6.5 and 6.6 respectively, at the beginning of $0 \mathrm{w}$ and 6.3 at the beginning of $2 \mathrm{w}$ but after the $4 \mathrm{w}$, the content of Enterobacter in the two groups became 6.4 and 6.7, the difference increasedy.
\end{abstract}

Key words: Lactobacillus plantarum, inflammatory bowel disease, intestinal flora disorder, bacterial translocation

In recent years, the incidence of Inflammatory Bowel Disease (IBD) has gradually increased worldwide and increased mortality, high incidence of complications and prolonged hospitalization often occur in patients with IBD with deep venous thrombosis. Even in areas with a relatively low incidence in the past, such as Asia, it has become more prevalent in recent years and its incidence shows a slow upward trend.

Whitlock et al. ${ }^{[1]}$ examined the effects of rifaximin $(10,30,50 \mathrm{mg} / \mathrm{kg} / \mathrm{d})$ and prednisolone $(10 \mathrm{mg} / \mathrm{kg} / \mathrm{d})$ on 2,4,6-trinitrobenzenesulfonic acid (TNBS) induced colitis in mice. He induced colitis in mice by rectal administration of TNBS and by histological scoring of clinical and colonic injury, Interleukin (IL)-2, IL-12, IL-12, IL-1, IL-2, IL-2, IL-1, IL-1, IL-2, IL-12, IL-1, IL, Interferon Gamma (IFN- $\gamma$ ) and Tumor Necrosis Factor-Alpha (TNF- $\alpha$ ) (protein and messenger RNA). Subsequently, Bei determined Immunoglobulin A $\left(\operatorname{IgA}^{+}\right)$and IgA-microbial composition by $16 \mathrm{~S}$

*Address for correspondence

E-mail: ran617008dizo@163.com----xe7033

November-December $2021 \quad$ Indian Journal of Pharmaceutical Sciences

November-December $2021 \quad$ Indian Journal of Pharmaceutical Sciences

Ribosomal RNA (rRNA) sequencing (IgA-SEQ), dividing the abundance of this component in the $\operatorname{Ig} \mathrm{A}^{+}$ component by that in the IgA- component, to estimate the relative IgA coating rate of each bacterial species, representing the IgA coating index. Although Bei's experiment is relatively perfect, it did not well exclude the influence of differences between individuals ${ }^{[2]}$. To investigate the effect of Lactobacillus plantarum (LP) on intestinal barrier function under inflammatory conditions, Lang et al. ${ }^{[3]}$ used IL-10 Knockout (IL-10 ${ }^{-/}$) mice as an inflammatory intestinal tract model disease, and IL-10 ${ }^{-/}$and Wild Type (WT) mice received LP or ringer solution for $4 \mathrm{w}$. Histological scores and clinical

This is an open access article distributed under the terms of the Creative Commons Attribution-NonCommercial-ShareAlike 3.0 License, which allows others to remix, tweak, and build upon the work non-commercially, as long as the author is credited and the new creations are licensed under the identical terms

Accepted 04 November 2021 Revised 03 March 2021

Received 05 April 2020 Indian J Pharm Sci 2021;83(6):1174-1180 
manifestations of colitis were observed. Intestinal paracellular permeability was measured by Ussing method. TNF- $\alpha$ and IFN- $\gamma$ were detected by EnzymeLinked Immunoassay (ELISA). Tight junction protein expression and distribution were measured by western blot and immunofluorescence, respectively. Lang et al. ${ }^{[3]}$ study lacks sufficient evidence in the confirmation of the results and it is difficult to achieve the real experimental results. Except for the normal control group, the other animals were given $30 \%$ Total Surface Area (TBSA) third degree burn $24 \mathrm{~h}$ after injury and then endotoxin $20 \mathrm{mg} / \mathrm{kg}$ was injected intraperitoneally. Ceftriaxone $60 \mathrm{mg} / \mathrm{kg}$ was injected intraperitoneally $24 \mathrm{~h}$ after the second impact in each group, twice a $\mathrm{d} 12 \mathrm{~h}$ apart and rhubarb $50 \mathrm{mg} / \mathrm{kg}$ was orally administered twice a d 12 $\mathrm{h}$ apart. The incidence of bacterial translocation in the intestinal flora, large intestine, liver, lung, mesenteric lymph nodes and blood in each group was detected on the $1^{\text {st }}, 3^{\text {rd }}$ and $9^{\text {th }} \mathrm{d}$ after treatment. Koç et al. ${ }^{[4]}$ research has insufficient data collection on experimental subjects, which may cause experimental errors.

Fifteen mice were selected and divided into three groups. Each group was gavaged with different bacterial solutions and buffers. The effects of LP on intestinal flora and bacterial translocation were measured at $0 \mathrm{w}$, $2 \mathrm{w}$ and $4 \mathrm{w}$ at the beginning of the experiment. The results were calculated and compared by one-way ANOVA to determine the benefit of probiotics on IBD.

Probiotics are native health-care strains, refer broadly to all substances that are applied to other animals or humans and can help the host improve the balance of microbes in the intestine $\mathrm{e}^{[5]}$. It contains many kinds of bacteria, such as Lactobacillus acidophilus North Carolina Food Microbiology (NCFM), Bifidobacterium bifidum (commonly known as B bacteria), Bifidobacterium animalis $\mathrm{Bb}-12$, yeast, LP, Lactobacillus lactis (commonly known as A bacteria), Lactobacillus bulgaricus, etc. These bacteria can help the intestinal tract to inhibit harmful bacteria, adjust the composition of colonies and enhance the ability of digestive tract. If the number of probiotics in the human body increases, then the number of harmful bacteria will be reduced accordingly. Therefore, the health of the human body is closely related to whether the original ecological balance of bacteria in the intestinal tract is balanced. Adding appropriate amount of intestinal probiotics or intestinal probiotic powder to milk can better protect the intestinal tract of infants and young children ${ }^{[6]}$.

The intestine is the largest micro ecological system and the largest immune organ of the human body and the human body interacts slowly with the outside through the gastrointestinal mucosa ${ }^{[7]}$. And people can promote the normalization of intestinal bacterial flora through various metabolic effects and their growth, which can reduce the production of putrefactive substances in the intestine and maintain the normal operation of intestinal function.

Lactose intolerance is a phenomenon of abdominal pain, abdominal distension and other adverse reactions in human body due to the lack of metabolic lactase after intake of some dairy products ${ }^{[8]}$. Signs of reduced lactase activity are evident in $75 \%$ of adults worldwide, with more than $90 \%$ of lactose intolerant patients in some Asian countries. Lactobacillus containing galactosidase activity can produce lactic acid to a large extent, reduce the concentration of lactose and facilitate human absorption and digestion.

After entering the intestinal tract, probiotics cannot only colonize the intestinal tract, keep the balance of intestinal microflora, but also directly act on the host's immune system, induce intestinal immunity and stimulate immune organs such as spleen and thymus and enhance the activity of macrophages ${ }^{[9]}$. It promotes $\mathrm{B}$ and $\mathrm{T}$ lymphocyte responsiveness to antigen stimulation. It can exert specific immune activity and further promote the enhancement of immune function of the body.

Some metabolites of tumor growth, such as lactic acid, bacteriocins and polysaccharides, can be inhibited by probiotics $^{[10]}$. By producing enzymes that inhibit the transformation of carcinogens, the body's immune system can be activated, especially the activity of B lymphocytes, Natural Killer (NK) cells, macrophages and the way in which cell mutations are inhibited. It can also reduce the $\mathrm{pH}$ value in the intestine, stimulate the peristalsis of the intestine, exclude carcinogens and pathogenic bacterial toxins in the intestine to the body and reduce the possibility of carcinogenesis.

$\mathrm{T}$ cells can produce a large amount of IL-12 after probiotic induction and IL-12 can have a good inhibitory effect on the production of $\operatorname{IgE}$ to prevent allergy ${ }^{[11]}$. Therefore, the more probiotics are present in the intestine of normal people, the less likely they are to develop allergic diseases.

Through assimilation, probiotics can inhibit the activity of cholesterol synthase (3-hydroxy-3-methylglutarate CoA reductase) and reduce the content of cholesterol. Probiotics can also co-precipitate cholesterol in food with hydrolyzed bile salts in the small intestine through 
bile salt hydrolase to help it be excreted in the form of feces.

Intestinal flora disorder plays an important role in the occurrence and development of obesity. The intake of appropriate amount of probiotics can effectively prevent and treat obesity.

First, increase satiety and suppress appetite: probiotics can stimulate the release of satiety factors such as Glucagon-Like Peptide-1 (GLP-1) and Cholecystokinin (CCK) and reduce the secretion of gastric growth stimulating hormone, thereby reducing food intake and reducing fat and weight accumulation. Second, lowering cholesterol: Probiotics can reduce the absorption of cholesterol by co-precipitation as well as assimilation. Third, adjust the intestinal microflora: after probiotics enter the intestine, increase Bacteroidetes, decrease Firmicutes, balance the intestinal microflora, decrease the permeability of intestinal epithelial cells, decrease the content of Lipopolysaccharide (LPS) in the circulation and reduce inflammatory factors, thereby improving the sensitivity of insulin.

Diabetic patients also have intestinal flora disorder. Probiotics can reduce the host's absorption of glucose and reduce blood glucose level by absorbing glucose and entering the body. By reducing the concentration of LPS in the circulation, probiotics can improve insulin sensitivity, reduce inflammation and enhance insulin resistance, thus achieving the goal of preventing diabetes.

Specific skin manifestations only occurs in patients with Crohn's Disease(CD), i.e., the intestinal pathology of CD is consistent with its histopathological manifestations, non-caseous granuloma with infiltration of dermal lymphocytes, multinucleated giant cells, eosinophils or plasma cells. Due to the different location of skin involvement, it can also be divided into skin metastatic $\mathrm{CD}$ and continuous skin manifestations.

Reactive skin manifestations are caused by abnormal immune responses caused by common antigens of skin and intestinal flora. Patients with Ulcerative Colitis (UC) and $\mathrm{CD}$ in IBD may have reactive skin manifestations. Mucosal auto-inflammation and innate immunity in IBD are closely related to such skin lesions. It mainly includes: aphthous stomatitis, gangrenous pyoderma, aphthous periadenitis, proliferative pyoderma pyoderma stomatitis, synovitis acne-pustulosis proliferative pyoderma, aseptic septic arthritis, Sweet syndrome, Bowel-Associated Dermatosis Arthritis Syndrome (BADAS), aseptic abscess syndrome, Hypertrophic-
Osteomyelitis (HOA) syndrome, Synovitis, Acne, Pustulosis, Hyperostosis, Osteitis (SAPHO) syndrome, Pyogenic Arthritis Pyoderma gangrenosum and Acne (PAPA syndrome), etc.

Relevant skin manifestations are associated with chronic inflammatory states of IBD and gene expression of specific Human Leukocyte Antigen (HLA). Relevant skin manifestations of IBD mainly include psoriasis, erythema nodosum, a variety of autoimmune bullous diseases (such as linear $\lg \mathrm{A}$ bullous disease, acquired epidermolysis bullosa), vasculitis, phlebitis, vitiligo, lichen planus, erythema multiforme, secondary amyloidosis, urticaria etc. Extremely rare include squamous cell carcinoma and Bowen's disease.

Secondary skin manifestations are closely related to drug-induced or nutrient deficiency. Zinc deficiency (enteropathic acrodermatitis) is more common and in $\mathrm{CD}$, enteropathic acrodermatitis has a higher incidence than UC.

The pathogenesis and etiology of IBD are still not clear, but the related inflammatory reaction caused by abnormal reaction of intestinal mucosal immune system has an important impact in the pathogenesis of IBD. At present, we believe that this is caused by the interaction of multiple factors, mainly including immune factors, genetics, environment and infection.

The incidence of IBD (UC or CD) caused by environmental factors has been increasing in recent decades. This phenomenon first appeared in economically and socially highly developed countries, first in Northern Europe, North America, then Southern Europe, Western Europe and most recently South America and Japan. This phenomenon reflects subtle but important changes in environmental factors, such as smoking, diet and other factors that are not fully defined.

Genetic factors, another important phenomenon of IBD incidence is the incidence of relatives. The incidence of first-degree relatives of IBD patients is significantly higher than that of the general population, but the incidence of spouses of IBD patients is no different from that of the general population. Among them, the incidence of $\mathrm{CD}$ in dizygotic twins is significantly lower than that in monozygotic twins. Because of the different genetic backgrounds and races of the population, the results of early studies on cytokine gene polymorphisms and HLA alleles are different. However, in recent years, through genome mapping and cloning, we found that chromosomes 3, 7, 12 and 
16 were susceptible points for IBD after comparing a large number of IBD family samples. IBD, on the one hand, is a polygenic disease, on the other hand, it is also a disease with genetic heterogeneity (different people can be caused by different genes). Under the action of certain environmental factors, patients will be affected by genetic susceptibility.

The role of microorganisms in the pathogenesis of IBD has been emphasized. Although no specific microorganisms with a constant relationship with IBD have been found, IBD (especially CD) may be caused by abnormal autoimmune response of normal intestinal flora. This is because, in IBD animal models, immunedeficient animals are created by knocking out genes or transgenic methods and intestinal inflammation does not occur in a sterile environment, but occurs in a normal flora state.

Microbiota is abundantly present in the body cavity and skin of human body. After a period of evolution, they can form interdependent, dynamic and balanced symbiotic relationship with the host. The most important and huge part of the human microbial system is the microflora in the gastrointestinal tract, which is an important link in the human body to absorb nutrients, develop diseases, digest food and so on. With the development of science and microbial detection technology, more and more people realize the importance of whether the microecological environment in the gastrointestinal tract is balanced in human health. At present, the research direction is mostly the relationship between diseases and intestinal flora and the gastrointestinal flora is affected by drugs.

Symbiotic and probiotic bacteria in the intestine form a bacterial membrane on the intestinal mucosa, which protects the body and also absorbs nutrients. Under normal conditions, the surface layer of intestinal mucosal flora is mainly Enterococcus and Escherichia coli, which belong to facultative anaerobes or aerobic bacteria and can swim freely. The middle layer of anaerobes of intestinal mucosal flora, in which Bacteroides is dominant, while the deep layer is a specific anaerobe, which is close to the upper wall of the mucosa and can form a fixed bacterial membrane structure after binding to the specific receptors of the surface layer. The acidic metabolites of these bacteria, mainly Lactobacillus and Bifidobacterium, have spectral antimicrobial components, which can effectively control the development of conditional pathogenic bacteria and pathogenic bacteria in the gastrointestinal tract, so that the bacteria in the growth process can produce as few harmful substances as possible and can also reduce endotoxin to accurately resist the invasion of the intestinal wall by passing bacteria.

However, if the bacteria in the gastrointestinal tract multiply too much, it will induce a variety of diseases, such as some bacteria will retrograde from the stomach to the throat and oral cavity, which will cause pneumonia. If the bacteria in the upper part of the small intestine multiply too much, they will also destroy digestive enzymes and decompose a large number of bile acids, resulting in poor digestion of the body. In addition, bacterial overgrowth can reduce nitrate to nitrite and one of the important factors that trigger gastric cancer is its synthesis of nitroso compounds. If the increase of bacteria in the small intestine will cause intestinal IBD, Clostridium difficile-associated diarrhea, irritable syndrome and so on, it will also produce a large number of harmful substances, which will cause the body to have a chronic toxic response after absorption in the ileum and jejunum. On the contrary, if the number of a certain flora in the gastrointestinal tract decreases, the antagonistic ability of organisms will be reduced, infections, constipation and diarrhea will occur, and may also make the colonization of foreign microorganisms or even change the intestinal flora, which cannot be reversed once changed.

\section{MATERIALS AND METHODS}

\section{Experimental subjects and materials:}

Experimental subjects: We purchased LP from infant feces isolated and purified from our hospital. Because of its good probiotic properties, we selected LP as the representative of probiotics in this experiment and selected fifteen $8 \mathrm{w}$ old female mice as experimental subjects and IL-10 $10^{-/}$mice as the model of IBD. We stored LP at $-20^{\circ}$ by freezing it at low pressure in bacterial powder, where the bacterial powder was diluted by Ringer buffer and cultured it with modified $\mathrm{MC}$ medium and $37^{\circ}$ anaerobically for $48 \mathrm{~h}$ within $2 \mathrm{~h}$ after dilution, so that its viable bacterial count was as high as $5 \times 10^{10} \mathrm{CFU} / \mathrm{g}$.

Experimental materials: The instruments and reagents used in the experiment include Ringer buffer, pancreatized soybean agar medium, TPY basic medium, anaerobic tank, modified MC medium, anaerobic air bag, N-N agar medium, VM-10 whirlpool oscillator, MacConkey plate, SW-CJ ultraclean workbench, blood agar culture plate, MicroscanAutoscan-4 bacterial identification system, HHS type constant temperature 
water bath, RA-ID plate, DNP-9028 constant temperature, temperature incubator, Scientz sterile homogenizer, TMQR-3250 pressure steam sterilizer, etc.

\section{Experimental steps:}

The IL-10 $0^{-/}+\mathrm{LP}$ group was given $0.5 \mathrm{ml} \mathrm{LP}$ bacterial solution $\left(1.0 \times 10^{9} \mathrm{CFU} / \mathrm{ml}\right)$ every $\mathrm{d}$, while the blank control group and IL-10 $0^{-/}$group were given $0.5 \mathrm{ml}$ ringer buffer solution for $4 \mathrm{w}$. Fecal samples were collected at 0,2 and $4 \mathrm{w}$ after the experiment and the changes of intestinal flora were detected. After the experiment, bacterial translocation of mesenteric lymph nodes and spleen was detected by sterile collection method.

Approximately $0.1 \mathrm{~g}$ of fresh mouse feces collected under sterile conditions were placed in Ringer buffer at $4^{\circ}$ and transferred to the microbial operating room within $1 \mathrm{~d}$. After shaking and mixing, the suspensions were diluted about 10 times. The appropriate dilutions were coated on modified MC (lactic acid bacteria), MacConkey medium (Enterobacteria), N-N medium (Clostridium perfringens), TPY medium (Bifidobacterium). All media except MacConkey medium were incubated anaerobically at $37^{\circ}$ for $48 \mathrm{~h}$, while MacConkey medium was incubated aerobically for $24 \mathrm{~h}$. Bacterial colony counts in all media require preliminary identification based on gram staining, microscopy and colony characteristics, which are then converted to bacterial content per gram of feces in $\log 10(\mathrm{CFU} / \mathrm{g})$.

\section{Treatment of experimental results:}

In this paper, the experimental results of Bifidobacterium, Lactobacillus, Clostridium perfringens and Enterobacter were analyzed by one-way ANOVA. One-way ANOVA was selected among all data analysis methods. The first step is to obtain the data structure of one-way ANOVA and then divide factor A into multiple levels by one-way ANOVA: $A_{1} \ldots A_{r}$, set the $X_{i j}$ survey index at each level as a whole. Then, it can also be assumed for the $\mathrm{R}$ population at $\mathrm{R}$ level: firstly, each population obeys the normal distribution, secondly, the homogeneity of variance also needs to be possessed by each population and finally, it must be ensured that these populations are pairwise independent and cannot have multiple linear relationships. If a sample of $\mathrm{N}$ volumes is obtained from each level of the assumed factor A and the sample has the desired variance 2 , then the mean is $I$ and the required normal population is $N\left(\mu_{i}, \sigma^{2}\right)$ $(\mathrm{i}=1,2,3 \ldots \ldots . \mathrm{r})$ and its calculation formula is as follows:
$\mathrm{X}_{\mathrm{ij}}=\mu+\mathrm{a}_{\mathrm{i}}+\varepsilon_{\mathrm{ij}}\left(\mathrm{i}=1,2, \ldots \ldots, \mathrm{r} ; \mathrm{j}=1,2, \ldots \ldots, \mathrm{n}_{\mathrm{i}}\right)(1)$

$\sum_{\mathrm{i}=1}^{\mathrm{r}} \mathrm{n}_{\mathrm{i}} \mathrm{a}_{\mathrm{i}}=0, \varepsilon_{\mathrm{y}} \sim \mathrm{N}\left(0, \sigma^{2}\right)(2)$

Among them, each epsilon $\varepsilon_{\mathrm{ij}}$ is independent of each other, although both $\mu$ and $\sigma$ are unknown values, but they exist independently at each level of factor A.

\section{REGULATION OF INTESTINAL FLORA BY LP}

\section{LP regulates intestinal bifidobacteria and lactobacilli in mice:}

The content of bifidobacteria in the LP liquid of the IL$10^{--}+\mathrm{LP}$ group increased after $2 \mathrm{w}$, but there was no significant difference compared with the IL-10 ${ }^{-/-}$group $(p>0.05)$. The regulatory effect of LP on intestinal bifidobacteria in mice is shown in fig. 1.

It can be seen from fig. 1 that the content of bifidobacteria in LP bacterial liquid increased significantly after 4 w compared with IL-10 ${ }^{-/}$group $(\mathrm{p}<0.05)$, but did not return to normal level compared with the blank control group ( $\mathrm{p}>0.05)$. The regulatory effect of LP on intestinal Lactobacillus in mice is shown in fig. 2.

Fig. 2 shows that before intervention, the content of lactobacilli in the intestine of mice in the IL-10 $10^{-/-}$group decreased significantly, the content of lactobacilli in IL-

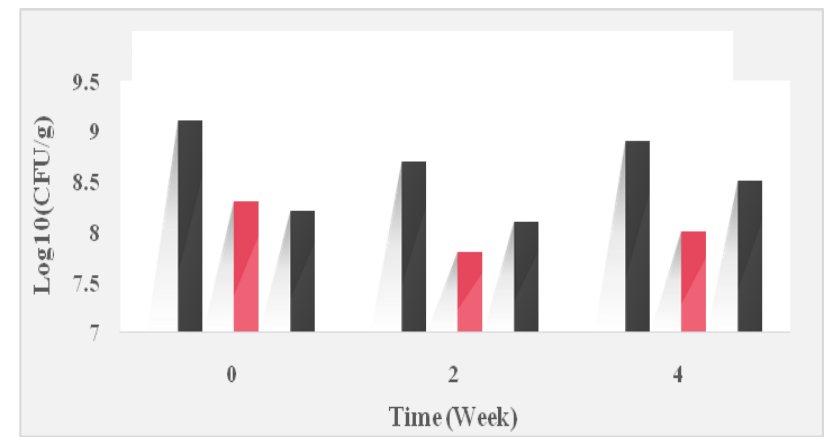

Fig. 1: Regulatory effect of LP on intestinal bifidobacteria in mice ( $\square$ Blank control group; ( $\square$ ) IL-10 ${ }^{-/-}$group; ( $\square$ IL$10^{--}+$LP group

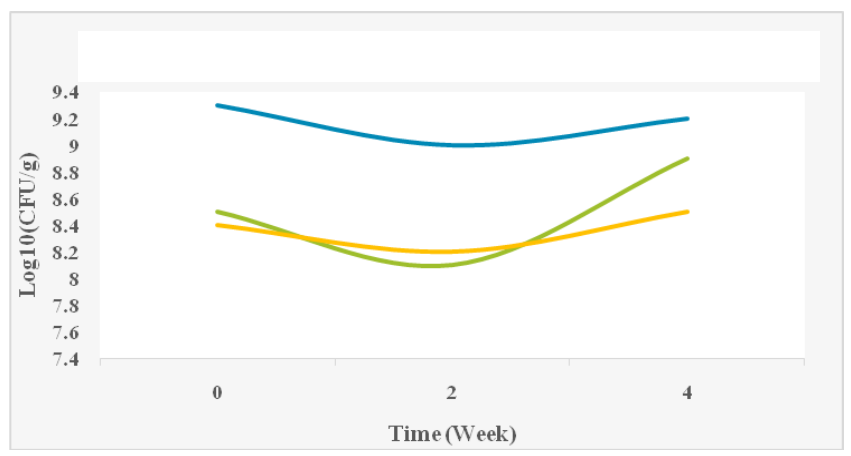

Fig. 2: Regulatory effect of LP on intestinal Lactobacillus in mice, ( $)$ Blank control group; ( $\mathrm{IL}^{-10^{-/-}}$group; ( $\mathbf{I L}^{-10^{-/}+\mathbf{L P} \text { group }}$ 


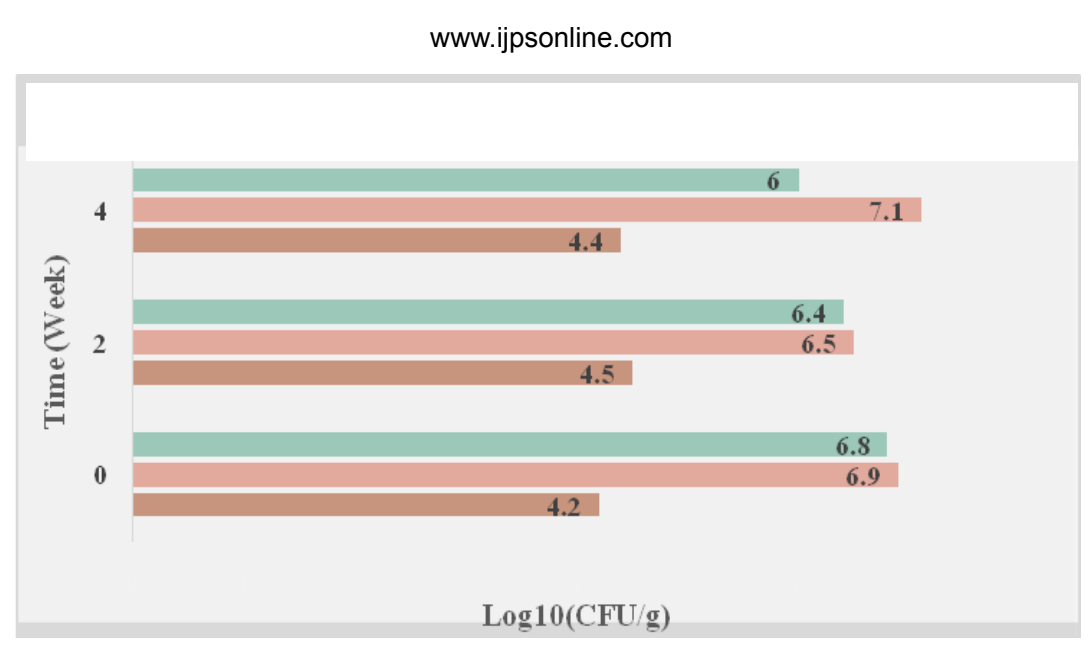

Fig. 3: Regulation of LP on intestinal Clostridium perfringens in mice, ( ) IL-10-/-+LP group; ( ) IL-10-/- group; ( $)$ Blank control group

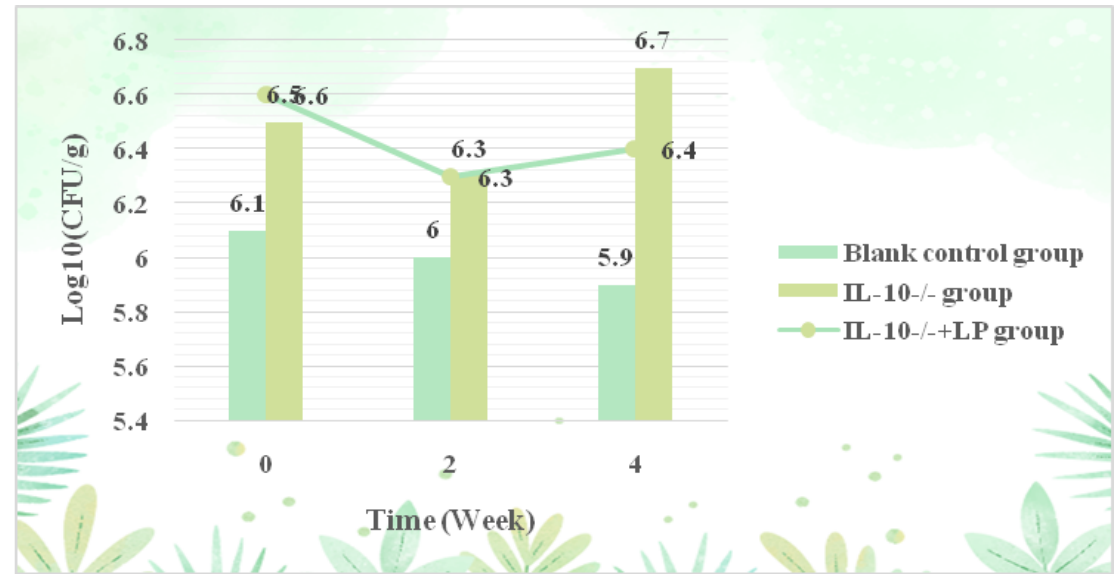

Fig. 4: Regulation effect of LP on intestinal enterobacteria in mice

$10^{-/}+$LP group increased significantly $(\mathrm{p}<0.05)$ after 2 $w$ of intragastric administration of LP bacterial fluid and there was no statistical difference between the content of lactobacilli in IL-10 $-1-$ LP group and blank control group after $4 \mathrm{w}$ of intervention ( $\mathrm{p}>0.05)$.

\section{Regulation of LP on Clostridium perfringens and enterobacteriaceae in mice:}

The regulatory effect of LP on intestinal Clostridium perfringens in mice was shown in fig. 3. According to the data in fig. 3 , the content of Clostridium perfringens in the IL-10-- 1 LP group was significantly lower than that in the IL- $10^{-/}$group after $2 \mathrm{~W}$ of administration $(\mathrm{p}<0.05)$. After $4 \mathrm{w}$ of intervention, the content of Clostridium perfringens also increased significantly, but did not return to the normal level $(\mathrm{p}<0.05)$. The regulatory effect of LP on Enterobacteriaceae in mice is shown in fig. 4.

It can be seen from fig. 4 that the content of Enterobacteriaceae in blank control group is the lowest at three time points, with an average of 6 , at the beginning of the experiment, the levels of Enterobacteriaceae in IL-10 $0^{--}+$LP group and IL-10 $0^{-/}$group were 6.5 and 6.6 respectively at the beginning of the experiment and were 6.3 after the first $2 \mathrm{w}$. However, the Enterobacteriaceae content of the two groups changed to 6.4 and 6.7 after the first $4 \mathrm{w}$.

\section{Regulation of LPon intestinal bacterial translocation:}

Probiotics are defined as non-pathogenic, living microorganisms that, when absorbed in sufficient quantities, can produce more beneficial effects on the body than nutrition. Probiotics can play a beneficial role in human health through a variety of ways, such as the regulation of intestinal flora, so probiotics can also be used as a means to regulate intestinal flora to treat many diseases related to flora imbalance. Among them, the regulatory effects of LP on bacteria translocation in mesenteric lymph nodes and spleen are shown in Table 1 and Table 2.

As shown in Table 1 and Table 2, no bacterial translocation was detected in the spleen and mesenteric 
TABLE 1: REGULATION OF LP ON TRANSLOCATED BACTERIA IN MESENTERIC LYMPH NODES

\begin{tabular}{|l|c|c|}
\hline Group & $\mathbf{n}$ & Mesenteric lymph node \\
\hline Blank control group & 5 & 0 \\
IL-10 $1 /$ group & 5 & $4.11 \pm 0.43$ \\
IL-10 $1 /+$ LP group & 5 & $2.43 \pm 1.40$ \\
\hline
\end{tabular}

TABLE 2: REGULATORY EFFECT OF LP ON TRANSLOCATED BACTERIA IN SPLEEN

\begin{tabular}{|c|c|c|}
\hline Group & $\mathrm{n}$ & Spleen \\
\hline Blank control group & 5 & 0 \\
\hline IL-10 $\%$ group & 5 & $5.54 \pm 0.66$ \\
\hline IL-10/-LP group & 5 & $3.67 \pm 1.81$ \\
\hline
\end{tabular}

lymph nodes of the control group mice after the end of the experiment, while the number of translocated bacteria in the mesenteric lymph nodes and spleen of the IL- $10^{-/}$group mice was significantly higher than that of the blank control group $(p<0.05)$.

Maintenance of the body's health has received a lot of attention in recent years and intestinal microecology plays a great role in it. If the intestinal microecology is unbalanced, it will induce a variety of diseases, such as non-alcoholic liver disease, diabetes, cancer and obesity. At present, there is no effective treatment for IBD, the main reason is that it is a recurrent, chronic autoimmune disease and the etiology is still uncertain. Probiotics have less toxic and side effects than other IBD treatments because of their obvious regulatory effects on various bacteria. Therefore, LP in probiotics was used as a representative to carry out the experiment. The experimental subjects were 15 female mice and the mice were used as animal models of IBD. The method of control experiment was used to compare the effects of probiotics.

We randomly divided them into three equal groups: blank control group, IL-10 $0^{-/}$group and IL-10 ${ }^{-/-}+$LP group and gavaged them with different bacterial solutions and buffers. Changes in the intestinal flora of mice were detected at $0 \mathrm{w}, 2 \mathrm{w}$ and $4 \mathrm{w}$ after the start of the experiment and bacterial translocation was recorded at the end of the experiment in mice with IBD. The detected intestinal flora included modified MC (lactic acid bacteria), MacConkey medium (Enterobacter), $\mathrm{N}-\mathrm{N}$ medium (Clostridium perfringens), TPY medium (Bifidobacterium) and the detection sites of bacterial translocation included mesenteric lymph nodes and spleen. After experimental comparison, we found that the content of modified MC (lactic acid bacteria) and TPY medium (bifidobacteria) decreased significantly and the bacterial translocation also increased. After intragastric administration of LP bacterial fluid for
$4 \mathrm{w}$, probiotics could regulate the intestinal tract of mice, balance the intestinal flora and reduce bacterial translocation.

Therefore, LP balances the intestinal flora by reducing "harmful bacteria" and increasing "beneficial bacteria", while also reducing bacterial translocation in the intestine, further enhancing the intestinal barrier function. However, there are still many difficulties to be applied in clinical practice, such as the dose of probiotics and the selection of strains, which still need a lot of experiments and scientific discussions.

\section{Conflict of interests:}

The authors declared no conflicts of interest.

\section{REFERENCES}

1. Whitlock SM, Enos CW, Armstrong AW, Gottlieb A, Langley RG, Lebwohl M, et al. Management of psoriasis in patients with inflammatory bowel disease: from the Medical Board of the National Psoriasis Foundation. J Am Acad Dermatol 2018;78(2):383-94.

2. Yue B, Ren YJ, Zhang JJ, Luo XP, Yu ZL, Ren GY, et al. Anti-inflammatory effects of fargesin on chemically induced inflammatory bowel disease in mice. Molecules 2018;23(6):1380.

3. Lang M, Rozalska A, Berry D, Riva A, Gasche C. Bacterial translocation into the mucus of crypts is associated with proximal colonic tumorigenesis in IL-10-/-xMSH2loxP/loxP Vill-cre (DKO) mice. J Gastroenterol 2016;54(5):44.

4. Koç TR, Tarhan ÖR, Sarıcık B. Effects of peritoneal lavage and dry cleaning on bacterial translocation in a model of peritonitis developed using cecal ligation and puncture. Ulus Travma Acil Cerrahi Derg 2018;24(4):281-6.

5. Q1 YP, Zhang T, Wang RP, Song YL, Duan GL. Effects of celastrol on serum bile acid metabolite profiling in mice with inflammatory bowel disease. Pharma Care Res 2018;18(6):42933.

6. Sheehan D, Shanahan F. The gut microbiota in inflammatory bowel disease. Gastroenterol Clin North Am 2017;46(1):14354.

7. Goto Y, Kurashima Y, Kiyono H. The Gut Microbiota and Inflammatory Bowel Disease. J Gannan Med Univ 2017;27(4):388.

8. Knackstedt R, Gatherwright J. The role of thermal injury on intestinal bacterial translocation and the mitigating role of probiotics: A review of animal and human studies. Burns 2020;46(5):1005-12.

9. Moharem HA, Fetouh FA, Darwish HM, Ghaith D, Elayashy $\mathrm{M}$, Hussein A, et al. Effects of bacterial translocation on hemodynamic and coagulation parameters during living-donor liver transplant. BMC Anesthesiol 2018;18(1):1-7.

10. Suk KT, Kim MY, Jeong SW, Jang JY, Jang YO, Baik SK. Impact of bacterial translocation on hepatopulmonary syndrome: a prospective observational study. Dig Dis Sci 2018;63(1):248-56.

11. Tsien C, Antonova L, Such J, Garcia-Martinez I, Wong F. Impact of bacterial translocation on sarcopenia in patients with decompensated cirrhosis. Nutrients 2019;11(10):2379. 